\title{
Methodology of Customized Bus Lines and Adjustment based on Soft Time Windows
}

\author{
ZhiJie Li ${ }^{1, a}$, Rui Song ${ }^{2, b}$ \\ ${ }^{1}$ MOE Key Laboratory for Urban Transportation Complex Systems Theory and Technology, Beijing \\ Jiaotong University, Beijing, 100044, China \\ ${ }^{2}$ School of Traffic and Transportation, Beijing Jiaotong University, \\ Beijing 100044, China \\ a12114248@bjtu.edu.cn, ${ }^{\text {brsong@bjtu.edu.cn }}$
}

\begin{abstract}
Keywords: Customized Bus; Vehicle Routing Problem; Soft Time Window
Abstract. An innovative mode of public transport(PT) service, known as customized bus(CB), has been springing up across China. This service, providing advanced, personalized and flexible demand-responsive PT, is offered to specific clientele, especially commuters. In order to resolve the problem of passenger personalized demand service and improve customer satisfaction, this paper focuses on constructing a methodology of customized bus lines and adjustment based on Soft Time Windows. Then the model is solved by the Cplex software. Taking the small-scale network as an example, different route strike of bus timetable could be obtained, and the results show that although the departure time is different, the access order is the same for the demand point. Due to the road resistance, there will be some waiting time for delay, which will guide the practical application.
\end{abstract}

\section{Introduction}

As travel demand is relatively consistent, traditional public transport is always short of service innovation, and service standards as well as transport capacity lack pertinence. Many original ground public transportation suffer a massive loss of passengers, regular bus in the middle and long distance travel has been at a disadvantage. Faced with the increasingly diversified travel needs, a single homogenizing conventional public transport services will inevitably produce low vehicle turnover, unbalanced load rate, unreasonable choice of vehicle models and other problems, resulting in high operating costs. The introduction of customized business bus not only meets the need of passenger personalized travel, but also guide more individuals to give up private car travel, which can ease traffic jams.

At home and abroad there are much related research on the school bus routing problem. Angel et al. [1] put forward the concept of "clustering structure after the first path" for the school bus routing problem without mixed load, and construct the vehicle routing. Euchi et al. [2] consider how to decide the school bus arrive the student stop, take the minimum distance of vehicle into a target, and study the city school bus routing problem in Tunisian city with the variable neighborhood search algorithm and ant colony optimization. Park et al. [3] learn the school bus routing problem with mix loading mode of stdents, and put forward the better algorithm, and prove the result with benchmark instance finally. Bennett B. T. et al. [4] take the total distance by bus as the goal in order to solve the school bus routing problem for single school and single vechicle model, and solve the problem of the size of the 256 depots with the improvement of C-W saving algorithm. Kim et al. [5] study the school bus routing problem with time windows, and solve that each different school can be serviced by a school bus for many times. Junhyuk et al. [6] develop a mixed load algorithm for the school bus routing problem (SBRP) and measure its effects on the number of required vehicles. Simchi-Levi et al. [7] claim that mixed load is allowed for the most part in New York city.

The above-mentioned domestic and foreign research did not give an overall consideration of time window, separation of loading and unloading, and open-loop VRP, most of which only consider two or three factors. However, these factors should be considered when solving the problem of real customized business bus route optimization design. Studies of vehicle routing both 
at home and abroad mainly focus on the improvement of models and algorithms. However, few studies have been done on VRP models to solve the problem of customized commercial bus routes. But with the popularization of customized business bus, The study of bus route is ofrealistic significance. Based on previous studies, this paper presents a further study on the customized business bus, and puts forward a solution to model with Cplex software.

\section{Problem description and a mathematical formulation}

Suppose that each bus only runs one round route and starts from depot and ends at the same place, and number of passengers at pickup and delivery stop is known, also only consider a single peak, namely morning peak or evening peak, not consider two-way peak at the same time, meanwhile the vehicle is driven under the ideal road condition, regardless of road resistance, and the depot is owned by the same company and can be freely used, at last there no passengers get on or get off at depot, passengers can on get on at pickup stop and get off at delivery stop;

Relevant parameter variable definition. The definition of symbol and implication used in the model is shown in Table 1:

Table 1 Relevant parameter variable definition

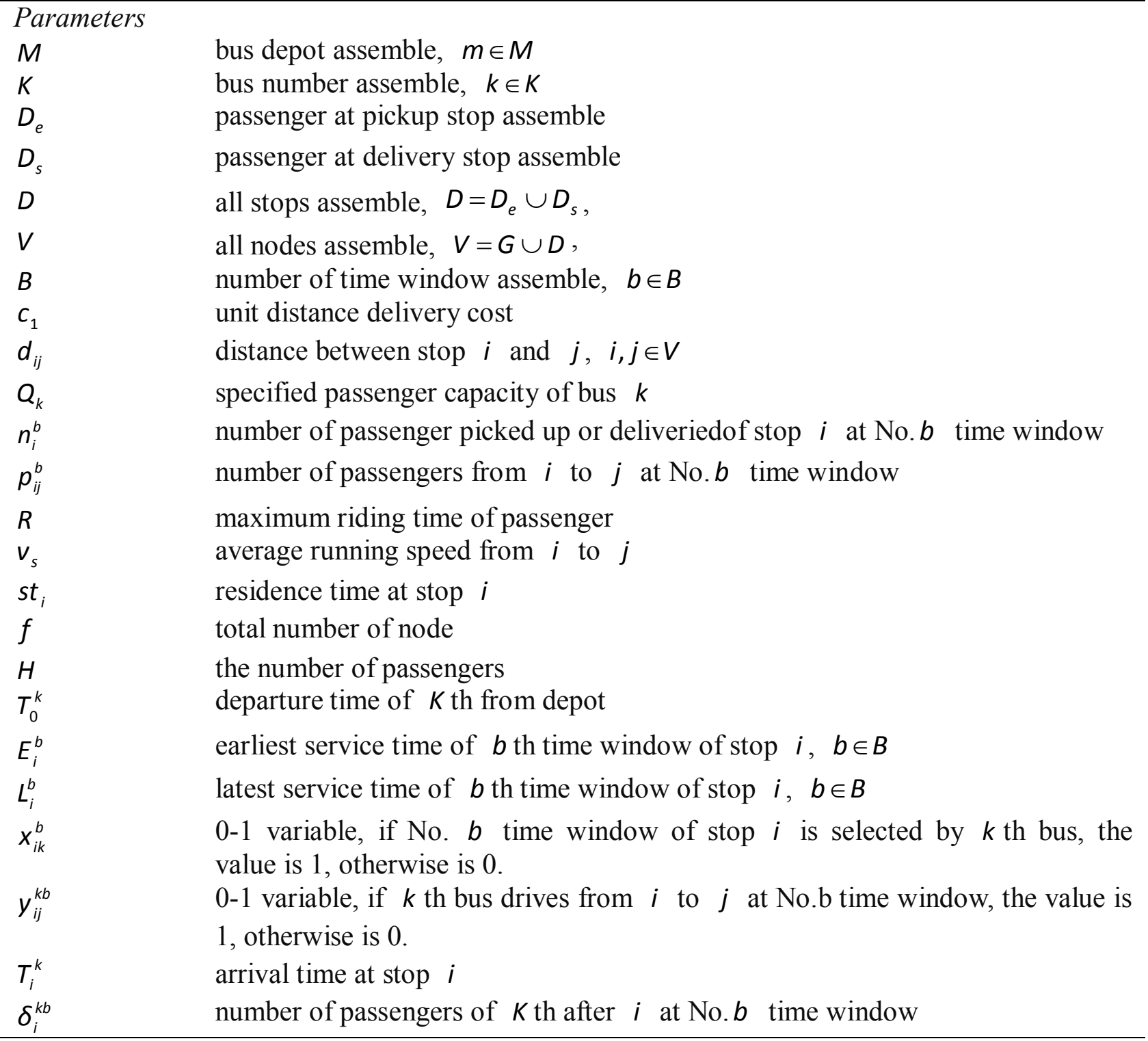

Route optimization of customized business bus with soft window. In order to minimize operation cost, the objective function is shown as: 
$\operatorname{Minf}=c_{1} \sum_{k \in K} \sum_{i \in V} \sum_{j \in V} \sum_{b \in B} d_{i j} y_{i j}^{k b}$

s.t.

$$
\begin{aligned}
& \sum_{k \in K} x_{i k}^{b} \geq 1, \quad i \in D, b \in B \\
& \sum_{i \in M} y_{i u}^{k b}-\sum_{j \in M} y_{u j}^{k b}=0, \quad \forall u \in D, k \in K, b \in B \\
& y_{i j}^{k b} \leq x_{j k}^{b}, \quad i \in D_{e}, j \in D_{s}, k \in K, b \in B \\
& T_{i}^{k}+y_{i j}^{k b}\left(s t_{i}+\frac{d_{i j}}{v_{s}}\right) \leq T_{j}^{k}, \quad i \in D, j \in D, k \in K, b \in B \\
& \sum_{i \in D} y_{i j}^{k b}=x_{j k}^{b}, \quad j \in D, j \neq i, k \in K, b \in B \\
& \sum_{j \in D} y_{j i}^{k b}=x_{i k}^{b}, \quad i \in D, i \neq j, k \in K, b \in B \\
& \sum_{i \in D} x_{i k}^{b} \leq f, \quad k \in K, b \in B \\
& \sum_{i=d(k)} y_{i j}^{k b}=\sum_{i=d(k)} y_{j i}^{k b}=1, \quad j \in D, k \in K, b \in B \\
& \sum_{i \in D_{e}} p_{i j}^{b} y_{i j}^{k b} \leq n_{j}^{b}, \quad j \in D_{s}, k \in K, b \in B \\
& \sum_{j \in D_{s}} p_{i j}^{b} y_{i j}^{k b} \leq n_{i}^{b}, \quad i \in D_{e}, k \in K, b \in B \\
& 0<T_{j}^{k}-T_{i}^{k} \leq R, \quad i \in D_{e}, j \in D_{s}, k \in K \\
& E_{i}^{b} \leq T_{i}^{k} \leq L_{i}^{b}, \quad i \in D, k \in K, b \in B \\
& T_{0}^{k}+\frac{d_{i j}}{v_{s}} \leq T_{j}^{k}, \quad i=m, j \in D_{e}, k \in K \\
& \sum_{i \in D_{e}} \delta_{i}^{k b} \leq Q_{k}, k \in K, b \in B \\
& \sum_{i \in D_{e}} \sum_{j \in D_{s}} p_{i j}^{b} y_{i j}^{k b} \leq \sum_{i \in D_{e}} \delta_{i}^{k b}, \quad k \in K, b \in B \\
& H_{i}^{k} \leq n_{i} \sum_{j \in D} y_{i j}^{k}, \quad i \in D_{e}, j \neq i, k \in K \\
& H_{i}^{k} \geq \sum_{j \in D} y_{i j}^{k}, \quad i \in D_{e}, j \neq i, k \in K \\
& x_{i}^{k} \leq H_{i}^{k}, \quad i \in D, k \in K \\
& \sum_{i \in D_{e}} \delta_{i}^{k b}=\sum_{i \in D_{s}} \delta_{i}^{k b}, k \in K, b \in B \\
& x_{i}^{k} \in\{0,1\}, \quad i \in D, k \in K \\
& y_{i j}^{k b} \in\{0,1\}, \quad i \in V, j \in V, k \in K, b \in B \\
& \delta_{i}^{k b} \geq 0, \quad i \in V, k \in K, b \in B \\
& T_{i}^{k} \geq 0, \quad i \in D, k \in K
\end{aligned}
$$

Constraints (2) ensure that each stop can be serviced by several buses within each time window, and each stop can be serviced. Constraints(3) ensure that each bus arrives at stop $i$ and leaves from stop i. Constraints(4) are the relationship between the route of the bus and the designated stop.Constraints(5) are the order of bus visiting constraints, ensuing that bus must first visit pickup 
stop before they can visit corresponding delivery stop. Constraints(6) and (7) show the relationship between the two variables. Constraints(8) ensure that the number of nodes served by bus is less than or equal to the total number of nodes.Constraints(9) insist that each vehicle must start from depot to pickup stop and come back to paking lot from delivery stop. Constraints(10) and (11) ensure that the number of passengers transported between the pickup stop and the delivery stop. Constraints (12) are time constraints from pickup stop to delivery stop. Constraints (13) ensure that the vehicle must arrive at the stop within each defined time window. Constraints (14) are the relation between departure time and arrival time. Constraints (15), (16), (17) and (18) are the capacity of the bus. Constrants (19) are the demand of the stops. Constraints (20) ensure that the number of passengers at the pickup stops is equal to the number of passengers at the delivery stops. Constraints (21) and (22) are $0-1$ constraints, limit value can only be 0 or 1 . Constraints (23) and (24) are nonnegative constraints, the limit value can take nonnegative number.

\section{Example}

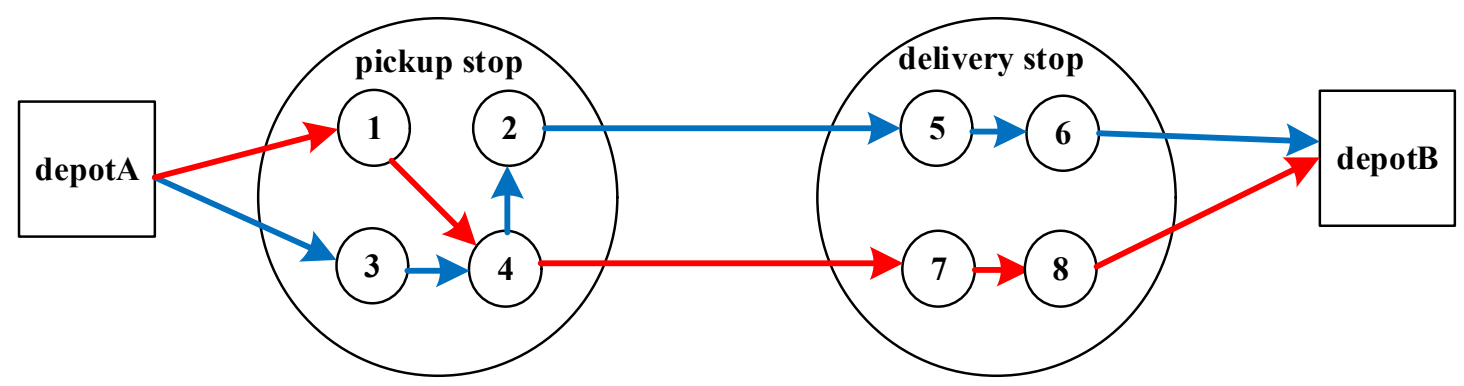

Fig.1. A network diagram

In order to describe the problem, we first construct a network diagram with two depots, four pickup stops and four delivery stops, total ten nodes, which is shown in Fig.1. Wherein, two depots are respectively depot $\mathrm{A}$ and depot $\mathrm{B}$, for convenience, we set four pickup stops as 1,2,3, and 4, four delivery stops as 5,6,7, and 8 . The passengers at four bus stops have different travel requirements for five delivery stops, such as the red line shown in Figure 1, indicating that the first bus departs from depot $\mathrm{A}$ and traverses pickup stop 1 and 4 respectively within the first time window specified by the stop, passengers at stop 1 and 4 are likely to get on and get off at stop 7 and 8 . After delivering all passengers, this bus will leave for depot B. Other route with different colors also have similar situation.

According to the model established above, we assign the parameters. We assume that there two depots and six buses. The unit distance cost $c_{1}$ is 10 yuan $/ \mathrm{km}$, the average speed of vehicle is 40 $\mathrm{km} /$ hour. The distance between nodes $d_{i j}$ is shown in Table 2. The rated passenger capacity of vehicle $Q_{k}$ is 55 . The number of passengers at stop i at No.b time window isshown in Table 3 . The maximum riding time is 3 hours, the waiting time of vehicle at stop i is 25 seconds. The earliest and the latest service time of No.b time window of stop $i$ are shown in Table 4.

Table 2. Distance between nodes

\begin{tabular}{ccccccccccc}
\hline stops & depotA & depot B & 1 & 2 & 3 & 4 & 5 & 6 & 7 & 8 \\
\hline depotA & 0 & 21 & 5 & 7 & 4 & 6 & 13 & 15 & 15 & 29 \\
depotB & 21 & 0 & 18 & 16 & 19 & 17 & 29 & 28 & 34 & 15 \\
1 & 5 & 18 & 0 & 4 & 3 & 5 & 16 & 17 & 18 & 26 \\
2 & 7 & 16 & 4 & 0 & 5 & 4 & 15 & 16 & 17 & 24 \\
3 & 4 & 19 & 3 & 5 & 0 & 4 & 13 & 14 & 16 & 26 \\
4 & 6 & 17 & 5 & 4 & 4 & 0 & 11 & 12 & 14 & 24 \\
5 & 13 & 29 & 16 & 15 & 13 & 11 & 0 & 5 & 7 & 21 \\
6 & 15 & 28 & 17 & 16 & 14 & 12 & 5 & 0 & 6 & 19 \\
7 & 15 & 36 & 18 & 17 & 16 & 14 & 7 & 6 & 0 & 21 \\
8 & 29 & 15 & 26 & 24 & 26 & 24 & 21 & 19 & 21 & 0 \\
\hline
\end{tabular}


Table 3 The number of passengers get on or get off at No.b time window (people)

\begin{tabular}{|c|c|c|c|c|c|c|c|c|}
\hline stop & 1 & 2 & 3 & 4 & 5 & 6 & 7 & 8 \\
\hline 1 & 46 & 72 & 86 & 74 & 70 & 81 & 73 & 54 \\
\hline$(2)$ & 62 & 74 & 70 & 82 & 82 & 86 & 52 & 68 \\
\hline
\end{tabular}

(note: in table 3 (1) indicates No.1 time window, (2) indicates No.2 time window)

Table 4 The earliest and the latest service time of No. $b$ time window of stop $i$

\begin{tabular}{|c|c|c|c|c|c|c|c|c|c|}
\hline stop & number & 1 & 2 & 3 & 4 & 5 & 6 & 7 & 8 \\
\hline \multirow{2}{*}{ the earest service time } & first & $7: 12$ & $7: 16$ & $7: 13$ & $7: 12$ & $7: 30$ & $7: 35$ & $7: 35$ & $7: 36$ \\
\cline { 2 - 10 } & second & $7: 27$ & $7: 30$ & $7: 28$ & $7: 27$ & $7: 45$ & $7: 50$ & $7: 50$ & $7: 51$ \\
\hline \multirow{2}{*}{ the latest service time } & first & $7: 22$ & $7: 27$ & $7: 18$ & $7: 24$ & $7: 37$ & $7: 40$ & $7: 46$ & $7: 48$ \\
\cline { 2 - 9 } & second & $7: 37$ & $7: 40$ & $7: 38$ & $7: 37$ & $7: 55$ & $8: 00$ & $8: 02$ & $8: 03$ \\
\hline
\end{tabular}

IBM Cplex 12.5 was used. The computational results under the configuration of i5-2410MCPU and 4G Ram, after about 4 seconds, the objective function got an optimal solution, the result is shown as table 5 .

Table 5 Solution of algorithm

\begin{tabular}{|c|c|c|c|}
\hline bus & number & visiting order of demand stop & delivery passengers (people) \\
\hline \multirow{2}{*}{1} & the first time & $1-2-3-4-5-6-7-8$ & 55 \\
\cline { 2 - 4 } & the second time & $1-2-3-4-5-6-7-8$ & 55 \\
\hline \multirow{2}{*}{2} & the first time & $1-2-3-4-5-6-7$ & 55 \\
\cline { 2 - 4 } & the second time & $1-2-3-4-5-6-8$ & 55 \\
\hline \multirow{2}{*}{3} & the first time & $4-5$ & 53 \\
\cline { 2 - 4 } & the second time & $4-5$ & 13 \\
\hline \multirow{2}{*}{4} & the first time & $2-5$ & 15 \\
\cline { 2 - 4 } & the second time & $2-5$ & 55 \\
\hline \multirow{2}{*}{5} & the first time & $3-4-8$ & 45 \\
\cline { 2 - 4 } & the second time & $3-4-8$ & 55 \\
\hline \multirow{2}{*}{6} & the first time & $2-6$ & 55 \\
\cline { 2 - 4 } & the second time & $2-6$ & 55 \\
\hline
\end{tabular}

We can see from table 5 that with different depature time, the six buses starting from depot 1 visit the different demand stop that is, but the routes are almost same, except the route of the 2th bus is $\mathrm{A} \rightarrow 1 \rightarrow 2 \rightarrow 3 \rightarrow 4 \rightarrow 5 \rightarrow 6 \rightarrow 7 \rightarrow \mathrm{B}$ at No.1 time window, and another route of the 2 th bus is $\mathrm{A} \rightarrow 1 \rightarrow 2 \rightarrow 3 \rightarrow 4 \rightarrow 5 \rightarrow 6 \rightarrow 8 \rightarrow \mathrm{B}$ at No.2 time window. This is because passengers reserve the route in advance through mobile APP. The route of customized business bus is fixed when the bus company confirms the route, which will not be changed by other demand. Therefore, the route of two vehicles is different. The passengers of 3th, 4th and 5th are different, because of delivery number is different for different time has different demand at each stop, and large coefficient of road impedancebetween starting stationswill cause time delayin some sections, resulting that vehicle can not reach corresponding demand stop on time.

\section{Conclusions}

In order to meet the needs of different passengers personalized travel on customized business bus, we optimize the route of customized business bus. In the traditional vehicle routing problem (VRP) base model, we consider the restriction of multiple time windows, regard customized business bus as a route selection problem under the restriction of multiple time windows, construct a customized 
business bus route optimization model based on multi-time windows, and solve the problem with Cplex. Taking the virtual network constructed by two depots and eight stops as examples, we get route under different departure times. Although the departure time is different, the order of visiting demand stops is the same. And because of the road resistance, there will be waiting time delay. This paper can make a good reference for practical customized business bus to plan route.

\section{Acknowledgements}

This research has been partially supported by the research project the National Natural Science Foundation of China (No.61374202).

\section{References}

[1] Angle R D, Caudle W L, Noonan R, et al. Management Science, 1972, 18(6):279-288.

[2] Euchi J, Mraihi R. Swarm and Evolutionary Computation, 2012, 2:15-24.

[3] Park J, Tae H, Kim B I. European Journal of Operational Research, 2012, 217(1): 204-213.

[4] Bennett B T, Gazis D C. Transportation Research, 1972, 6:317-325.

[5] Kim B I, Kim S, Park J. European Journal of Operational Research, 2012, 218(2): 577-585.

[6] Junhyuk Park, Hyunchul Tae, Byung-In Kim. European Journal of Operational Research, 2012,217:204-213.

[7] Simchi-Levi, D. Chen X, Bramel J. A case study: School bus routing.2005,Springer, New York, pp.319-335. 